\title{
Treatment Outcome of Oncologic Patients Treated in the Emergency Department at Black Lion Specialized Hospital, Ethiopia
}

Fasil Sisay ${ }^{1}$, Sofia Kebede ${ }^{2}$, Asmamaw Abebe ${ }^{2}$, Fikrte Woldeselassie ${ }^{1}$, Girum Sebsibie $^{2}$ and Teklemariam Gultie ${ }^{3^{*}}$

${ }^{1}$ Menelik II Health Science College, Addis Ababa, Ethiopia

${ }^{2}$ College of Health Sciences, Addis Ababa University, Addis Ababa, Ethiopia

${ }^{3}$ Department of Midwifery, College of Medicine and Health Sciences, Arba Minch University, Ethiopia

*Corresponding author: Teklemariam Gultie, Department of Midwifery, College of Medicine and Health Sciences, Arba Minch University, Ethiopia; E-mail: tekledb2002@gmail.com

Received date: August 22, 2018; Accepted date: September 13, 2018; Published date: September 22, 2018

Copyright: ( 2018 Sisay F, et al. This is an open-access article distributed under the terms of the Creative Commons Attribution License, which permits unrestricted use, distribution, and reproduction in any medium, provided the original author and source are credited.

\begin{abstract}
Background: The emergency department is a section of an institution that is staffed and equipped to provide rapid and varied emergency care, especially for those who are stricken with sudden and acute illness or who are the victims of severe health problem. Oncology patients in the emergency department are causing a significant burden for health care system due to the late seeking of care by the cancer patient to the Hospital at an advanced stage.
\end{abstract}

Methods: A cross-sectional study was conducted at the black lion Specialized Hospital. A one-year data was abstracted of 426 cancer diagnosed patients. The extraction was done from April 1 to April 30, 2017. Data abstraction format was developed based on the nature of the record.

Result: Four hundred twenty-six oncology patients record were analyzed. The mean age was 42.5 years. Gastrointestinal cancer $128(30 \%)$ was the most common cancer type followed by Hematologic $81(19 \%)$ cancer. Based on symptoms the common complaints were a pain (14.8\%), Easily Fatigability $(11.7 \%)$, Dysphagia $(11.5 \%)$ and Bleeding (10.8\%).

Conclusions: The common types of cancer diagnosed at the emergency department were Gastro-intestinal and Hematology malignancies. The pain was the predominant complain among oncology patient present in an emergency department. Most of the cancer patients present in the emergency department are due to the effect of the tumor, one-tenth of the patient died while receiving care.

Keywords: Oncology; Pattern; Outcomes; Length of stay

\section{Introduction}

Cancer is one of the leading causes of morbidity and mortality all over the world. According to WHO report, there are about 14 million new cases were reported in 2012. Globally cancer is a second leading cause of death and 8.8 million deaths were recorded in 2015 that means about 1.6 deaths is due to cancer. Cancer causes an estimated $70 \%$ in low and middle-income countries the major reason for high mortality in the low-income country could be a delay in presentation, Unable to get diagnosis and treatment. In 2015 report indicated, only $35 \%$ of low-income country patients and more than $90 \%$ of highincome countries have access to treatment [1].

Oncological emergency care concern is including overcrowding, long waiting time and lack of access to specialized care. Cancer patient also visited frequently Emergency Department (ED) when they are near to end life with late stages of cancer. Cancer patients in the emergency department help to recognize life-threatening presentation but not directly related to care delivered in the emergency department. The cancer care quality issue is such as inadequate access and utilization for prevention, diagnosis service inadequate and uncoordinated care or fragmented health care delivery, poor symptom management monitoring and controlling of complication [2].
As malignancy is a common problem of the world and cancer patients also at high risk developing a wide range of medical emergencies. The medical emergency can present as a result of local tumor effect of rapid metastasis and may appear secondary to antineoplastic treatment. An oncologic emergency can be broadly classified into four categories (Metabolic, Hematologic, Structural and Treatment-Related Emergency). 5\% to 10\% incidence rate with Hypercalcemia, this problem should be corrected as much as early in ED. Almost all cancer patients ED visits are due to acute Tumor Lysis Syndromes and Lactic Acidosis [3]. Tumor Lysis is one of lifethreatening clinical condition most commonly encounters ED Physician and Nurses. Patients with $>80 \%$ Hematologic malignancies have a chance to develop Febrile neutropenia, which is eligible for acute care and even for admission. Leukocytosis is one of serious oncologic emergency in which mortality risk is more than $40 \%$ if untreated early. Spinal Cord Compression also the commonest ED presentation among oncology Neurologic emergency patient. Superior Vena Cava (SVC) obstruction is a common problem, which presents with chest pain, distended neck, and facial swelling. Another problem of the oncology patient that need emergency care is due to increased ICP, urine obstruction, anaphylaxis, and others [4]. Therefore, many conditions related to malignancy and their treatment can be life threating and need a very comprehensive and urgent emergency care. 
In Spain from 112 patients, most common cause to seek emergency care was 21 patients with Fever, 15 patient's Dyspnea acute Abdominal pain and pain about 11 patients; this all patients need admission and emergency care [5]. According to World cancer declarations progress report in 2016, Ethiopia cancer country profiles reported that mortality of Male is 14,500 and Female were 26,200 [6].

A study done in black lion specialized hospital oncology departments stated that $10 \%$ of patients didn't come early to Hospital. About 47\% was Gynecological Malignancies, 26\% Breast cancer, 22\% Head and Neck cancer, Sarcoma 15\%, GI 12\%, Hematologic and Urologic 9\% and Thyroid 5\%. As this study shows to us, as far as Oncology patient presentation is very late they will need frequency care to ED [7]. Emergency patient length of play has a significant role on emergency service and for patient life quality. Therefore, it is important to assess Treatment outcome of Oncologic Patients treated in the Emergency Department.

\section{Materials and Methods}

\section{Study area and period}

This study was conducted at the Black lion specialized hospital, Adult Emergency Department from April 2016 to March 2017. It is the largest referral hospital and the only oncologic treatment center in the country. The emergency department provides emergency care for more than 1847 emergency patients per month every year. This department has more than 56 beds, 62 Nurses and about 6 emergency Physicians working permanently. The hospital provides service in Internal Medicine, Surgical, Pediatric, Gynecological and Obstetrics, Oncology. This hospital provides a tertiary level referral treatment and it is opened for 24 hours for emergency services.

\section{Study design}

A cross-sectional study was conducted on oncology patients in black lion hospital adult Emergency department from April 01, 2016 to March 31, 2017.

\section{Population}

The study populations were all emergency patients who received treatment in the Black lion specialized hospital emergency department during the study period. All individuals above 13 years old and admitted with oncologic problems were included in the study. Those with incomplete medical records were excluded from this study.

\section{Sample size determination}

Since the objectives of this study are to determine the treatment outcome of oncology patients in adult ED for one year the records reviewed of all adult oncology patient admitted to the ED during April 01, 2016 to March 31, 2017.

\section{Data collection tools and procedures}

The data collection instrument was pre-tested for necessary modifications in the questionnaire. The questionnaires were prepared in the English language, as far as am using it to collect from the secondary data source. The collected data were compared and checked for completeness, missed questions by the supervisors. Two supervisors and five data collectors who have previous work experiences in data collection were recruited. They were trained and oriented for one day on the contents of the questionnaire and how to collect information from secondary data sources.

\section{Data analysis}

The quantitative data were entered by using, computer processing, and SPSS software version 20.00. The data description methods were by using proportion rates, percentages, ratios, Frequency distributions tables.

\section{Ethical Approval}

This study was conducted after obtaining ethical approval from the ethical review board of college of health sciences, Addis Ababa University. A written letter was presented to the emergency department from the college of health sciences. The clinical director was informed about the purpose of the study and confidentiality of the clients' information. After a thorough discussion with the clinical director, letter of support was written for the record and archive officer. The record and archive officers were informed about the study by the data collectors.

\section{Result}

Most of the patients were at age of 25 to 49.9 years old. The death rate is higher in the age of 50-74.9 years as compared to the age below 25 years. Majority of the patients admitted in the hospital for less than 10 days. Most of the patients were married (Table 1).

\begin{tabular}{|c|c|c|c|}
\hline \multirow[b]{2}{*}{ Variables } & \multirow{2}{*}{$\begin{array}{l}\text { Response } \\
\text { categories }\end{array}$} & \multicolumn{2}{|c|}{ Treatment outcome } \\
\hline & & Death & Alive \\
\hline \multirow[b]{4}{*}{ Age in years } & $1-24.9$ & $5(7.7 \%)$ & $60(92.3 \%)$ \\
\hline & $25-49.9$ & $23(11.2 \%)$ & $183(88.8 \%)$ \\
\hline & $50-74.9$ & $21(14.6 \%)$ & $123(85.4 \%)$ \\
\hline & $75-99.9$ & 0 & $11(100.0 \%)$ \\
\hline \multirow[b]{5}{*}{ Region } & Addis Ababa & $19(15.0 \%)$ & $108(85.0 \%)$ \\
\hline & Oromia & $14(10.5 \%)$ & $119(89.5 \%)$ \\
\hline & Amhara & $3(6.3 \%)$ & $45(93.8 \%)$ \\
\hline & SNNPR & $5(9.6 \%)$ & $47(90.4 \%)$ \\
\hline & Others* & $8(12.1 \%)$ & $58(87.9 \%)$ \\
\hline \multirow{2}{*}{$\begin{array}{l}\text { Length of stay in the } \\
\text { hospital }\end{array}$} & $1-10$ days & $46(12.1 \%)$ & $333(87.9 \%)$ \\
\hline & Above 11 days & $3(6.4 \%)$ & $44(93.6 \%)$ \\
\hline \multirow[b]{2}{*}{ Sex of patient } & Male & $23(10.8 \%)$ & $189(89.2 \%)$ \\
\hline & Female & $26(12.1 \%)$ & $188(87.9 \%)$ \\
\hline \multirow{4}{*}{$\begin{array}{l}\text { Marital status of the } \\
\text { patient }\end{array}$} & Married & $33(11.9 \%)$ & $245(88.1 \%)$ \\
\hline & Single & $12(10.7 \%)$ & $100(89.3 \%)$ \\
\hline & Windowed & $1(12.5 \%)$ & $7(87.5 \%)$ \\
\hline & Divorced & $3(10.7 \%)$ & $25(89.3 \%)$ \\
\hline
\end{tabular}


Citation: Sisay F, Kebede S, Abebe A, Woldeselassie F, Girum S, et al. (2018) Treatment Outcome of Oncologic Patients Treated in the Emergency Department at Black Lion Specialized Hospital, Ethiopia. J Integr Oncol 7: 213. doi:10.4172/2329-6771.1000213

Page 3 of 4

*Tigray, Somalia, Dire Dawa, Afar, Benishangul Gumuz, Gambella and Harari

Table 1: Socio-demographic characteristics of the patients and their treatment outcome in the black lion specialized hospital emergency department.

\section{Types of diagnosed cancer}

Gastro-intestinal cancer was the leading cause of death followed by gynecologic cancer. The least cause of death was skin cancer followed by urinary tract cancer (Table 2). The common chief complaints of the patients at the time of hospital visit were pain followed by fatigue and bleeding (Table 3).

\begin{tabular}{|c|c|c|c|}
\hline & & \multicolumn{2}{|c|}{ Treatment outcome } \\
\hline \multicolumn{2}{|l|}{ Variables } & Dead & Survive \\
\hline & Head and Neck & $4(9.3 \%)$ & $39(90.7 \%)$ \\
\hline & Gastro-intestinal & $13(10.2 \%)$ & $115(89.8 \%)$ \\
\hline & Breast & $3(14.3 \%)$ & $18(85.7 \%)$ \\
\hline & Gynecologic & $10(20.0 \%)$ & $40(80.0 \%)$ \\
\hline & Skin & 0 & $1(100.0 \%)$ \\
\hline & Urinary & $1(2.5 \%)$ & $39(97.5 \%)$ \\
\hline & Lung & $6(24.0 \%)$ & $19(76.0 \%)$ \\
\hline & Lymphoma & $4(23.5 \%)$ & $13(76.5 \%)$ \\
\hline & Hematologic & $7(8.6 \%)$ & $74(91.4 \%)$ \\
\hline & Sarcoma & $1(8.3 \%)$ & $11(91.7 \%)$ \\
\hline Diagnosed cancer & Others* & 0 & $8(100.0 \%)$ \\
\hline
\end{tabular}

*pancreatic cancer, thyroid cancer, prostate cancer

Table 2: Comparisons based on the type of diagnosed cancer between dead and survive in black lion specialized hospital emergency department.

\begin{tabular}{|c|c|c|c|}
\hline \multirow{3}{*}{$\begin{array}{l}\text { Variable } \\
\text { Chief complain of the patient }\end{array}$} & \multicolumn{2}{|l|}{ Treatment outcome } & \multirow{3}{*}{$\begin{array}{l}\text { Survive } \\
61(96.8 \%)\end{array}$} \\
\hline & \multicolumn{2}{|l|}{ Dead } & \\
\hline & \multirow{2}{*}{$\begin{array}{l}\text { Pain } \\
\text { Fatigability }\end{array}$} & \multirow{2}{*}{$\begin{array}{l}2(3.2 \%) \\
8(16.0 \%)\end{array}$} & \\
\hline & & & $42(84.0 \%)$ \\
\hline & $\begin{array}{l}\text { Neck and Face } \\
\text { Swelling }\end{array}$ & 0 & $17(100.0 \%)$ \\
\hline & Neurologic Symptoms & $1(5.6 \%)$ & $17(94.4 \%)$ \\
\hline & Dysphagia & $6(12.2 \%)$ & $43(87.8 \%)$ \\
\hline & Dyspnea & $9(23.7 \%)$ & $29(76.3 \%)$ \\
\hline & Fever & $2(8.3 \%)$ & $22(91.7 \%)$ \\
\hline & Infection & $8(88.9 \%)$ & $1(11.1 \%)$ \\
\hline & Nausea and Vomiting & $8(21.1 \%)$ & $30(78.9 \%)$ \\
\hline
\end{tabular}

\begin{tabular}{|l|l|l|l|}
\hline & Bleeding & $1(2.2 \%)$ & $45(97.8 \%)$ \\
\cline { 2 - 4 } & Others* & $4(5.4 \%)$ & $70(94.6 \%)$ \\
\hline *cough, constipation, rectal bleeding, breast lump, frequent urination
\end{tabular}

Table 3: A comparison based on the chief complaints at the time of admission between dead and survives in the black lion specialized hospital emergency department.

\section{Discussion}

The main complaints of oncologic patients were a pain (14.8\%). This finding is in line with a study finding in Australia (13\%), Spain (22.5\%), Italy (28.9\%) and Korea (19\%) in which Pain is the first and common symptom. Other report shows in Italy and USA Minneapolis Pain is the second complain [8-12]. This study demonstrates Bleeding (10.9\%), Nausea and Vomiting (8.9\%), and Fever (5.6\%) is a common complaint by patients. A similar report shows in Germany, Australia, and Switzerland [13].

Other study shows the length of stay of oncology patients in the Emergency Department is 2 hours a report in Germany, Australia and Switzerland [13]. When we compare with Australia the range was from minutes to an hour (57 minutes to 40 hours) median 6.8 hours [9]. In Taiwan and Cairo oncology patient length of stay in the Emergency Department is shorter than the black lion hospital emergency department, 2.91 hours and 3.6 hours [14,15]. According to the above study, other countries have a very short length of stay of oncology patients when compared with our study which accounts for the majority one to ten days.

Among 426 patients, $81 \%$ have a solid tumor and $19 \%$ of them have Hematologic malignancies. A Korean study showed $88.8 \%$ solid tumor and $11.2 \%$ Hematologic malignancies emergency [12]. Solidity and Hematologic emergency malignancies are nearly equal number. The Nigerian report [16] shows $54.6 \%$ of them are hematologic malignancies and $45.4 \%$ of them are solid malignancies. In Victoria, Australia research report shows 58\% solid tumor and $42 \%$ Hematologic [9]. Form the above reports different countries report shows higher frequency on solid malignancies, which is the same as our study findings.

About $30 \%$ is gastrointestinal cancer (Esophageal, Gastric, and Anorectal) recorded in black lion specialized hospital. A research in Australia has reported only 10.5\% (Upper GI, Digestive tumor, and Colorectal) this result shows gastrointestinal cancer in high when compared with this study. The other report from Turkey showed $25.4 \%$ is gastrointestinal cancer, this shows less result than this study but Taiwan study reports $34.8 \%$, this is higher than the current study. GI cancer is the first common cancer type seen in the black lion specialized hospital, but in Iran and Turkey, it is in second place, in Australia fourth-place $[17,18]$.

Gynecologic cancer is third cancer seen in our study; it accounts for $11.7 \%$, and other studies 6th cancer type in Canada. In Australia report also put Gynecologic cancer on the fifth place [9]. This shows gynecologic emergency cases are more common in black lion hospital than in other countries.

The emergency department patient outcome of Australian research report discovered that $4.6 \%$ death and $4.5 \%$ discharged to home. This study has less death than the current study [9]. This could be due to the 
Citation: Sisay F, Kebede S, Abebe A, Woldeselassie F, Girum S, et al. (2018) Treatment Outcome of Oncologic Patients Treated in the Emergency Department at Black Lion Specialized Hospital, Ethiopia. J Integr Oncol 7: 213. doi:10.4172/2329-6771.1000213

Page 4 of 4

fact that standard treatment is not well organized in the current study setting.

\section{Conclusion}

In black lion specialized hospital emergency department Gastrointestinal tract, Hematology, Gynecology, Head and Neck, Urinary, Breast, Lung cancer are the most common cancer diagnosed from April 2016 to March 2017. Lymphoma, sarcoma, and skin are rare cancer diagnosed in the hospital. The common symptoms of cancer patient present within the hospital were pain, dysphagia, fatigability and bleeding complained by a cancer patient. Dyspnea, Fever, Head and neck swelling, Neurologic symptoms and nausea/vomiting are rare symptoms complained by the patient. Regarding the treatment outcome of oncologic patients, 49 were dead and 377 were survived. As most of the patients seek health care at later stage it is important to increase their health seeking behavior and reduce the mortality rate. Further study should be conducted with a larger sample size to identify the factors associated with the survival status of the oncologic patients.

\section{References}

1. www.who.int/mediacenter/factsheet/75287/en/

2. Spinks TE, Gonzalez CE (2016) Quality measures for oncologic emergency medicine: In Oncologic Emergency Medicine, Springer Cham. $13-41$.

3. Rumi K, Sangeetha PW (2013) An overview of oncologic emergency, what family, physicians and their parents need to know: La Prensa Medical Argentina. 99: 10.

4. Silvo A, Juan M, Armenta E, Agel Herrera G (2015) Tumor lysis syndrome in the emergency department: challenge and solutions. Open Access Emerg Med 7: 39-44.

5. Kirstie G, Yann H, Nadita K, Mariam M, Rebecca M, et al. (2016 )World cancer declaration progress report 2016: tomorrow is now our journey 2025.

6. Cheryl H, Sisay T, Nazanin M, Christopher M, Lisa P (2015) Patterns and predictors of early mortality among emergency department patients in Addis Ababa, Ethiopia. BMC Res Notes 8: 605.

7. Wondimagegnehu T, Abera M, Aynalem A, Mathwose A (2015) Pattern of Cancer in TASH Oncology Center in Ethiopia from 1998 to 2010. Int J Cancer Res Mol Mech 1.
8. Swenson KK, Rose MA, Ritz L, Murray CL, Adlis SA (1995) Recognition and evaluation of oncology-related symptoms in the emergency department: Ann Emerg Med 26: 12-17.

9. Livingston PM, Craike M, Slavin M (2012) Clinical and economic burden of emergency department presentation for neutropenia following out the patient's chemotherapy for cancer in Victoria, Australia. The Oncologist 17: 998-1004.

10. Numico G, Cristofano A, Mozzicafreddo A, Cursio OE, Franco P, et al. (2015) Hospital admission of cancer patients: avoidable practice or necessary care? Plos One (10): e0120827.

11. Pajares M, Tobena A, Ferrer S, Serrona M, Vidal D, et al. (2009) Fever in cancer patients as a cause of attendance in emergency room. J Clin Oncol 27.

12. Ahn S, Lee YS, Lim KS, Lee JL (2012) Emergency department cancer unit and management of oncologic emergencies: experience in Asan Medical Center. Support Care Cancer 20: 2205-2210.

13. Elmar C, Irinaeus A, Normann W, Wrich S, Oliver M (2008) Radiotherapy in oncological emergencies--final results of a patterns of care study in Germany, Austria and Switzerland. Acta Oncological Journal 47: 81-89.

14. Yi Hui L, Danchen Chu, Nan-Ping Y, Chien-Lung C, Shun Ping C, et al. (2015) Emergency visits among end-of-life cancer patients in Taiwan: a nationwide population-based study. BMC Palliat Care 14: 15.

15. Alsitay SA, Raheem AA, Al-Zahrani AS, Mohammed AA, Sherisher MA, et al. (2016) Emergency department visits at the end of life of patients with terminal cancer: pattern, causes, and avoidability. Am J Hosp Palliat Care 33: 658-662.

16. Adewuyi SA, Ajekigbe AT, Campbel OB, Mbibu NM, Oguntago AO, et al. (2012) Pattern of oncologic emergency seen in adult cancer patient attending the Radiotherapy and Oncology Center, Ahmadu Bello University, teaching hospital, Zaria-Nigeria. Niger Postgrad Med J 19: 208.

17. Muallaoglu S, Karagun O, Besen Ali, Sezer A, Ozyilkan O (2014) Attribute of cancer patient admitted to the emergency department medicine. World J Emerg Med 5: 85-90.

18. Bahram M, Kambiz N, Elham SR, Mohammad F (2016) Epidemiology and death related factor of oncology patient in emergency department: Emergency 4: 145. 